TRANS $\cdot$ núm. $24 \cdot 2020$

MISCELÁNEA $\cdot 335-352$

Los programas de formación de traductores a nivel universitario a menudo se organizan en torno a asignaturas de traducción práctica en varias combinaciones lingüísticas y direcciones. Por norma general, cada asignatura se centra en una combinación lingüística y una dirección, y una de las características implícitas de esta organización es que el docente debe ser un hablante nativo de la lengua de destino. Este artículo tiene dos objetivos: de una parte, señalar las claves que permiten explicar la deslegitimación de la traducción A-B desde una perspectiva teórica; de la otra, presentar los resultados de un estudio exploratorio que analiza la perspectiva de un grupo de traductores en formación acerca de la direccionalidad a través del grupo de discusión como técnica de recogida de información.

PALABRAS CLAVE: direccionalidad de la traducción, traducción A-B, formación de traductores, traductor en formación, docente de traducción.

\title{
Estudio exploratorio-descriptivo sobre la direccionalidad en traducción: perspectiva del traductor en formación
}

SARA HorCAS-RuFlán

Universidad de Granada

\section{An Exploratory-Descriptive Study on Directionality in Translation: the translator trainee's perspective}

Most university translation programmes are usually structured around translation practice modules in different translation fields, and in various language combinations and directions. Standard practice is for each module to focus on one language combination and one direction. Likewise, one of the implicit assumptions of this teaching model is that teachers should be native speakers of the target language of the class. This paper pursues two goals. First, it highlights some of the key issues that allow to explain the delegitimisation of translation A-B from a theoretical standpoint; secondly, it describes the findings of an exploratory study that used the qualitative research methods of focus groups to explore directionality from the perspective of translator trainees.

KEY WORDS: directionality in translation, translation A-B, translator training, translator trainees, translator trainer. 


\section{INTRODUCCIÓN}

Actualmente suele aceptarse la especificidad de la denominada traducción «inversa» tanto en la práctica profesional como en la formación de traductores (Cómitre Narváez, 2003; Cruz Trainor, 2004; Pavlović, 2010; Wimmer, 2011; Gallego Hernández, 2014a). Dicha especificidad, se manifiesta no solo en las particularidades del proceso de traducción, sino también en factores relacionados con el mercado laboral y la situación de cada país, donde determinadas combinaciones lingüísticas exigen que los traductores profesionales deban desenvolverse con frecuencia en «inversa».

Sin embargo, de acuerdo con Hurtado Albir (2001: 57), durante mucho tiempo esta dirección ha atraído menos la atención de los teóricos. Y, si bien a lo largo de las dos últimas décadas se ha producido un incremento en el número de trabajos que se ocupan de esta cuestión (Beeby Lonsdale 1996; Campbell, 1998; Grosman et al., 2000; Kelly et al., 2003; Pokorn 2005, 2009, 2016; Contreras García y Turrión, 2013), sigue siendo manifiesta la controversia existente en la literatura especializada contemporánea.

Por otro lado, según ha apuntado Prunč (2003), puesto que la didáctica de la traducción tiene - $\mathrm{O}$ en todo caso debe tener- por objeto la reflexión crítica de la cultura de la traducción, sería lógico asumir que la clase de traducción es asimismo una de las fuentes primarias de creación y difusión de las «normas, convenciones, expectativas, valores socialmente establecidos, que son compartidos por todos los actores implicados en el proceso de traslación». Y, análogamente, Kelly (2003) señala que la literatura publicada sobre la formación de traductores se ha centrado en torno a los procesos y actividades en detrimento de los agentes informantes, sea este el caso de los docentes de traducción o de los traductores en formación.
En respuesta a dichas aseveraciones, el presente artículo tiene dos objetivos: de una parte, señalar las claves teóricas que permiten explicar la deslegitimación de la traducción «inversa» desde una perspectiva teórica; de la otra, explorar la direccionalidad en traducción a partir del análisis de un caso con traductores en formación del Grado de Traducción de la Universidad de Granada en el que se utilizó el grupo de discusión, una técnica de investigación cualitativa que resulta especialmente conveniente para identificar los valores atribuidos por un grupo social a un fenómeno determinado.

\section{DIRECCIONALIDAD: PERSPECTIVA TEÓRICA}

En los estudios dedicados a las teorías contemporáneas de la Traducción, especialmente en Occidente, el término «direccionalidad» alude a la sucesión de uso de los dos códigos lingüísticos que intervienen en todo proceso traslativo; es decir, la lengua «extranjera» y la «lengua materna» del traductor o intérprete. Asimismo, y sin perjuicio de lo anterior, existen en el debate de la direccionalidad otras posibles direcciones, como la traducción entre «lenguas extranjeras» o la traducción entre «lenguas maternas». No obstante, la literatura traductológica ha tratado la cuestión de la direccionalidad mediante el binomio traducción «directa» e «inversa», por lo que para dilucidar la evolución de esta cuestión es necesario revisar las menciones de la traducción «inversa» (en adelante, traducción $\mathrm{A}-\mathrm{B}^{1}$, por oposición a la traducción «directa» (i.e., tra-

I Adoptamos la terminología propuesta por el grupo de investigación AVANTI de la Universidad de Granada que se inspira en el sistema de clasificación de las lenguas de trabajo (A, B y C) de la Asociación Internacional de Intérpretes de Conferencia (AIIC), también utilizado en los ámbitos profesional y académico. 
Tabla 1. Terminología de la direccionalidad en varias lenguas (Beeby Lonsdale, 2009: 84)

DIRECCIONALIDAD DE LA TRADUCCIÓN

Traducción B-A

Traducción a la lengua materna

Traducción a la lengua de uso habitual

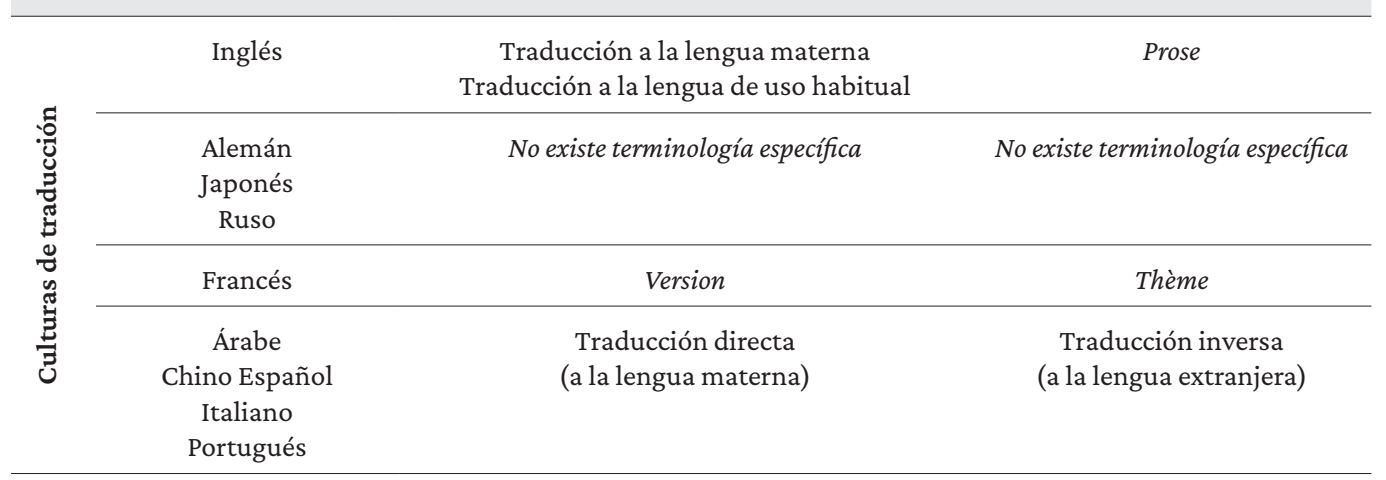

Traducción A-B ducción B-A), en la bibliografía existente (Kelly et al., 2003).

Como objeto de estudio, la investigación de la traducción A-B ha favorecido la reflexión crítica sobre la superioridad de la direccionalidad B-A desde una perspectiva teórica. Sin embargo, la miríada terminológica utilizada para referirse a la direccionalidad del proceso traslativo, a las lenguas de trabajo implicadas y a los usuarios de estas es representativa del estado naciente en el que permanece el debate científico en torno a esta cuestión. En este sentido, Beeby Lonsdale (2009: 84) se expresa en lo relativo a la ausencia de consenso en la terminología usada para referirse a la direccionalidad. A modo de ejemplo práctico, en la Tabla 1 recogemos las manifestaciones terminológicas correspondientes a varias lenguas, citadas por la autora en la Enciclopedia Routledge de Estudios de Traducción.

Esta variedad —o, en su caso, vacío- terminológico-conceptual refleja la existencia de un conjunto de normas y convenciones de traducción, según las cuales se establecen diferentes valores sociales y rangos de aceptación de formas de traducción. Podemos asumir que los términos generados por la literatura para referirse a la traducción A-B, considerada por muchos traductólogos como la «Cenicienta de los Estudios de Traducción» (Beeby Lonsdale, 1996: 100), constituyen un afán de perpetuar la subordinación de esta modalidad a la traducción B-A, la dirección legítima y natural. En este sentido, destaca el uso del término "inversa" (que se define como contrario o de sentido opuesto) para referirse a la traducción A-B, que contrasta con el discurso traductológico de países como Alemania, Japón o Rusia, en cuyas culturas de traducción la dirección A-B es ampliamente practicada.

Sin embargo, basta con realizar un breve recorrido por la historia universal de la práctica de la traducción, cuyo objetivo es responder a las preguntas: ¿qué se ha traducido?, ¿por quién?, ¿de qué manera?, y ¿en qué contexto social o político? (Woodsworth, 1998: 101) para descubrir que este no es un caso excepcional: instituciones políticas y religiosas, así como culturas y comuni- 
338 dades periféricas con lenguas minoritarias ${ }^{2}$, han apadrinado la traducción A-B como instrumento de difusión cultural. La Septuaginta, versión en griego del Antiguo Testamento, traducida del hebreo por un grupo compuesto por setenta y dos judíos eruditos (Robinson, 1997: 5); la Vulga$t a$, texto bíblico oficial de la Iglesia romana católica, que supone la traducción de la Biblia hebrea y griega al latín después de la caída del Imperio romano; o la traducción moderna de la Biblia a lenguas vernáculas (Nida y Taber, 1982), constituyen algunos ejemplos.

Sin embargo, a pesar de ser parte inequívoca de la práctica profesional de la traducción, el estudio de la traducción A-B ha sido ampliamente desatendido por la literatura traductológica. De acuerdo con Opdenhoff (2011: 4), el debate científico en torno a la direccionalidad permanece en un estado naciente, por lo que la investigación en este campo específico es imprescindible «si la ciencia quiere salir de una situación en la que los argumentos se basan más bien en posturas subjetivas e ideológicas que en hechos científicamente probados».

No obstante, en las últimas décadas se ha producido un incremento notable en el número de trabajos que se ocupan de este tema, como lo demuestra la celebración de dos jornadas monográficas en 1997 en Ljubljana (Grosman et al., 2000) y en 2002 en Granada (Kelly et al., 2003), que recogen algunas de las contribuciones más señaladas del panorama actual de la investigación en esta línea. Entre ellas destacan las aportaciones a la direccionalidad y la didáctica de la traducción (Beeby Lonsdale, 1996; Kelly, 1997; Martínez Melis, 2001; Zimmermann, 2007; Roiss, 2008; Wimmer 2011; Rodríguez y Schnell, 2012; Gallego Hernández, 2014b). Asimismo,

\footnotetext{
2 Para una descripción sobre las lenguas minoritarias, véase Branchadell (2012).
}

también se han llevado a cabo estudios de recepción de literatura traducida hacia el inglés como lengua B (Pokorn, 2005), estudios centrados en las diferencias entre el proceso de traducción B-A y A-B (Pavlović y Jensen, 2009; Alves Ferreira, 2012; Chodkiewicz, 2016; Ferreira et al., 2016; Whyatt, 2019), y estudios de mercado (Roiss, 2001; Pavlović, 2007; Pietrzak, 2013; Ličko, 2014; IAPTI, 2015). Más recientemente, Lima Fonseca (2015) y da Silva et al. (2017) han iniciado una nueva línea de investigación que indaga sobre el impacto de la direccionalidad en los procesos de edición y posedición.

\section{Las culturas de la traducción en la formación en traducción A-B}

La cultura de traducción (del alemán, «Translationskultur») supone uno de los principales factores que determinan la actitud prescriptiva frente a la teoría y práctica de la traducción. Este concepto, introducido al debate traductológico por Prunč (1997), se construye a través de la existencia de un subsistema desarrollado históricamente y formado por un conjunto de normas, convenciones, expectativas y valores socialmente establecidos, que son compartidos por los actores implicados en todo proceso traslativo. Por ello, cada cultura de traducción está incuestionablemente ligada a parámetros sociológicos, discursivos y normativos, que pueden colisionar, y que establecen, de forma más o menos dogmática, las formas de traducción consideradas erróneas. De este modo, encontramos identidades, como la anglófona ${ }^{3}$, en las que el veto a la traducción A-B forma parte del código deontológico de la profesión (ITI, 2012); así como minorías lingüísticas, como la nórdica, en las que

\footnotetext{
3 Véase el círculo de Krashen (1985) para una excelente explicación de esta problemática en la comunidad anglófona.
} 
existen razones de índole pragmática, relacionadas con el mercado profesional, para contemplar la traducción A-B en los planes de estudios.

En España, donde los estudios de Traducción e Interpretación han experimentado una creciente demanda, manifiesta en la vertiginosa multiplicación de los centros que ofrecen programas de grado y postgrado, los contenidos de los planes de estudios se han adaptado paulatinamente para conciliar las demandas de un mercado profesional cada vez más globalizado y flexible en el que la traducción A-B es una práctica frecuente (Roiss, 2001; Rodríguez-Inés, 2008; Gallego Hernández, 2014a).

La necesidad de formar a profesionales polivalentes, capaces de abordar esta forma de traducción, fue refrendada por el marco legal de la educación superior en España con la aprobación y entrada en vigor del Real Decreto 1385/1991, que incluyó la traducción A-B entre los contenidos de las materias troncales de los antiguos planes de estudios conducentes a la obtención del título universitario oficial de Licenciado en Traducción e Interpretación. Sin embargo, la armonización de las enseñanzas y títulos universitarios oficiales exigida por el proceso de convergencia europea, iniciado en 1999 con la Declaración de Bolonia, ha supuesto la sustitución paulatina de las licenciaturas por los actuales títulos oficiales de Grado en Traducción.

En este sentido, el Libro Blanco del Título de Grado en Traducción e Interpretación -que constituye una propuesta no vinculante sobre aspectos fundamentales del diseño del título de Graduado- reconoce entre los objetivos de formación la «competencia traductora general y especializada, tanto hacia la lengua propia como hacia al menos dos lenguas extranjeras», pero la traducción A-B ya no constituye una materia o contenido de obligatoria inclusión en los nuevos planes de estudios. Esto significa que las institu- ciones formativas, ya sean públicas o privadas, poseen libertad para decidir qué papel otorgar a la traducción A-B en el diseño de sus títulos.

A este respecto, una revisión de los planes de estudios realizada durante el curso 2017-2018 (Horcas-Rufián y Kelly, en prensa), nos ha permitido identificar que aproximadamente el 20 $\%$ de los planes de estudios a nivel de Grado no ofrece ningún crédito ECTS en traducción A-B. De hecho, solo 5 de los 28 existentes ofrecen más de 15 ECTS en esta dirección, el mínimo recomendado por el Libro Blanco para cada lengua extranjera y dirección de traducción. A nivel de máster solo 4 planes de estudios (de los 25 activos) ofrecen entre 1 y 7 ECTS de un total de 60 . Si contrastamos estas cifras con el estudio realizado por Wimmer (2011: 50) sobre la traducción A-B en los antiguos planes de estudio de traducción, cuyos datos señalan que la dirección A-B solía constituir entre el $25 \%$ y el $50 \%$ de las asignaturas de traducción, encontramos que la dedicación actual es notablemente inferior.

\section{El docente de traducción y el «hablante nativo»}

Además de los cambios de carácter institucional y académico a los que ya nos hemos referido, la configuración del Espacio Europeo de Educación Superior (EEES) ha propiciado el desarrollo de un debate profundo sobre la actualización de los fundamentos y los principios pedagógicos que orientan la acción docente a favor de un nuevo paradigma que pone el énfasis en los estudiantes como protagonistas del proceso de aprendizaje, el aprendizaje autónomo, la adquisición de competencias genéricas y específicas y la evaluación continua de los aprendizajes. En este contexto, el proceso de renovación metodológica podría verse inevitablemente truncado por la pleitesía a una figura docente concebida como centinela de la verdad traductológica, única conocedora 
340 de la traducción «correcta» (Kiraly, 1995: 99). La descripción de House de la clase tradicional de traducción nos sirve para ilustrar este escenario pedagógico:

The teacher of the course, a native speaker of the target language, passes out a text [...]. The text is then prepared, either orally or in a written form, for the following sessions and then the whole group goes through the text sentence by sentence, with each sentence being read by a different student. The instructor asks for alternative translation solutions, corrects the suggested versions and finally presents the sentence in its final «correct» form... This procedure is naturally very frustrating for the students. (House, 1980, 7-8, cit. Kiraly, op.cit.: 7).

Si bien este modelo tradicional podría pensarse superado en la formación de traductores profesionales, capaces de traducir hacia sus lenguas de trabajo activas (lengua A y B), constatamos que el diseño de los planes de estudios de las enseñanzas oficiales de Grado no solo continúa estableciendo claras diferencias con respecto a los objetivos y los contenidos académicos dependiendo de la dirección del proceso traslativo, sino también a los enfoques metodológicos adoptados. Sobre este particular, House (op. cit.) recoge en su primera línea una verdad incuestionada durante mucho tiempo que debería ser objeto de análisis y reflexión crítica: la asignación de la docencia tomando como premisa principal la lengua A del docente de traducción. Concretamente en España, Kelly (2005) pone de manifiesto que la necesidad de incluir la traducción A-B en los planes de estudios de las diferentes instituciones formativas ha llevado a la contratación de hablantes «nativos» de diferentes lenguas.

En una interesante visión crítica del estado de la formación de traductores en la universidad española, Mayoral (2001) señala que concretamente en la Facultad de Traducción e Interpretación de la Universidad de Granada, donde se ha establecido una separación estanca entre traducción B-A y traducción A-B, esta política lingüística sigue siendo manifiesta: el docente, especializado para ambas ramas y lenguas principales diferentes, debe ser «nativo» de la lengua de destino de la clase. Independientemente del posible debate sobre la conveniencia de esta medida, con demasiada frecuencia se asume de manera concluyente y unánime que el hablante nativo de una lengua está naturalmente capacitado para orientar y ayudar a los traductores en formación a desarrollar las competencias necesarias para poder desempeñar la traducción como actividad profesional. Sin embargo, este modelo heredado, que podría parecer razonable puesto que disipa cualquier duda sobre la competencia lingüística del docente, nace del precepto que niega a los hablantes «no nativos» de una lengua la capacidad para adquirir una competencia comunicativa completa ${ }^{4}$.

No obstante, el panorama descrito hasta ahora en torno a la autoridad del hablante nativo en el ámbito académico y profesional discurre por derroteros significativamente diferentes en muchas de las instituciones formativas que ofertan enseñanzas conducentes a la obtención de un título universitario oficial de Grado o Máster en Traducción en el EEES. Si bien es cierto que actualmente no existen estudios publicados que revelen datos sobre quién desempeña esta labor, con qué titulación, con cuánta experiencia profesional como traductor, o con qué formación docente, dar respuesta a esta demanda se ha convertido en un reto, especialmente en aquellos países o regiones que utilizan lenguas

\footnotetext{
4 Véase Davies (2003) para una discusión profunda sobre este tema.
} 
minoritarias o de difusión limitada, donde la traducción A-B es una práctica habitual; esto se traduce en una carencia notable de traductores profesionales de las lenguas más demandadas en los diferentes mercados de la traducción y de docentes de traducción con perfiles lingüísticos similares; véanse, a modo de ejemplo, Pokorn (2009) y Hagemann (2019) sobre direccionalidad y docencia.

En relación con esto, nos cuestionamos: ¿fomenta el perfil del hablante nativo la superioridad de la traducción B-A en la clase de traducción $A-B$ ?, ¿ se utilizan diferentes estrategias didácticas dependiendo de la dirección del proceso traslativo?, ¿constituye la lengua A del docente un factor clave para el éxito de la formación de traductores? Estas son algunas de las preguntas que sirvieron de punto de partida en un estudio empírico cuyo propósito fue explorar y describir la perspectiva de los traductores en formación sobre las implicaciones de la direccionalidad para la clase de traducción.

\section{ESTUDIO EXPLORATORIO-DESCRIPTIVO: PERSPECTIVA DEL TRADUCTOR EN FORMACIÓN}

Como punto de partida de este estudio exploratorio, utilizamos el grupo de discusión. La técnica del grupo de discusión se basa en la producción de discursos de un grupo en el que están representadas todas las personas relevantes de una comunidad, consiguiendo así una representación estructural que refleja la dinámica de una realidad (normas, valores, interacciones sociales, percepciones de una realidad, etc.) (Buendía et al., 1998: 263). De acuerdo con nuestro objetivo, a diferencia de otros métodos de interacción grupal en los que se busca consenso o decidir entre varias alternativas, como por ejemplo los procesos delphi o las técnicas de brainstorming, el grupo de discusión se ajusta satisfactoriamente a nuestras necesidades.

\section{Decisiones muestrales}

Desde un enfoque cualitativo, el muestreo adopta la forma de una selección de casos o informantes a través de un procedimiento intencional y deliberado que supone la implementación de un diseño de muestreo no probabilístico. Según McMillan y Schumacher (2005: 446), la selección de los sujetos comienza con una descripción de las cualidades o perfil deseado de las personas que poseen conocimiento sobre el tema en cuestión. En este caso, nuestra intención es representar estructuralmente al grupo social que se estudia, de forma que todas las posiciones respecto del tema de investigación estén presentes. De hecho, el grupo de discusión se caracteriza por ser homogéneo, pero conservando al mismo tiempo una heterogeneidad suficiente para permitir un contraste de opiniones (Krueger, 1988: 96). Según Valles (2003: 91), el criterio de heterogeneidad, entendido como un compromiso entre variación y tipicidad, resulta fundamental para el muestreo cualitativo: en los grupos de discusión no se persigue la representación estadística, sino la representación tipológica.

Otro rasgo de esta técnica de entrevista grupal es el número de informantes. Tradicionalmente un grupo de discusión ideal está compuesto por entre cinco y diez personas (Suárez Ortega, 2005: 26). El tamaño está condicionado por dos factores: debe ser lo suficientemente pequeño como para que todos tengan la oportunidad de exponer sus puntos de vista y lo suficientemente grande como para que exista diversidad en los mismos (Krueger, 1988: 34). No obstante, cada vez son más comunes los grupos de discusión reducidos (de entre cuatro y seis informantes). Las razones que subyacen a dicha tendencia son dos: 
Tabla 2. Composición del grupo de discusión deseado

\begin{tabular}{cccc}
\hline N.o informantes & Lengua A & Lengua B & Curso académico \\
\hline 1 & $\begin{array}{c}\text { Lengua mayoritaria } \\
\text { (inglés/francés/alemán) }\end{array}$ & Inglés/español & $\begin{array}{c}\text { Estudiante de } \\
\text { intercambio }\end{array}$ \\
\hline 1 & $\begin{array}{c}\text { Lengua minoritaria } \\
\text { (p. ej., neerlandés/esloveno/danés/...) }\end{array}$ & Inglés/español & $\begin{array}{c}\text { Estudiante de } \\
\text { intercambio }\end{array}$ \\
\hline 1 & Árabe & Español & $4^{\text {o }}$ \\
\hline 1 & Español & Inglés & $4^{\text {o }}$ \\
\hline 1 & Español & Francés & $4^{\text {o }}$ \\
\hline
\end{tabular}

reclutar y acomodar un grupo reducido es más fácil y resulta más grato para los informantes. En la Tabla 2 se exponen las características del grupo de discusión que integraría la representación tipológica de la población atendiendo a ambos aspectos y al propósito del estudio.

Una vez definidas las características de los informantes, dimos comienzo al proceso de contacto y selección. Puesto que la Ley Orgánica 15/1999 prohíbe expresamente la difusión de datos personales que permitan la identificación de una persona física si no existe previo consentimiento de la misma, como estrategia para localizar a los informantes se recurrió al reclutamiento sobre el terreno. Para ello, contactamos con los docentes responsables de las asignaturas de traducción A-B de diferentes lenguas B de la Facultad de Traducción e Interpretación de la Universidad

Tabla 3. Composición del grupo de discusión que participó en el encuentro

\begin{tabular}{ccccc}
\hline Sujeto & Nacionalidad & Lengua A & Lengua B & $\begin{array}{c}\text { Curso de plan de } \\
\text { estudios }\end{array}$ \\
\hline S1 & España & Español & Alemán & $3^{\text {o }}$ (cursando TA-B de 40) \\
\hline S2 & Alemania & Alemán & Inglés & $\begin{array}{c}\text { Estudiante de } \\
\text { intercambio }\end{array}$ \\
\hline S3 & España & Árabe y español & Inglés & $4^{\text {o }}$ \\
\hline S4 & España & Español & Inglés y francés & $4^{\text {o }}$ \\
\hline S5 & España & Español & Francés e inglés & $4^{\text {o }}$ \\
\hline S6 & Alemania & Alemán & Español e inglés & Estudiante de \\
& & & & intercambio
\end{tabular}


Tabla 4. Temas abordados en el grupo de discusión

Direccionalidad: implicaciones para la práctica de la traducción

Direccionalidad y traducción: la cuestión de la calidad.

Autoevaluación de la competencia traductora A-B.

Direccionalidad y autoeficacia.

La direccionalidad en la clase de traducción

Organización de la formación en traducción A-B/B-A en el plan de estudios.

Identificación de metodologías didácticas.

El perfil del docente de traducción

Relevancia de la lengua A del docente para la clase de traducción.

Competencias del docente de traducción.

La formación en traducción A-B

Satisfacción con la formación recibida.

Otras observaciones

de Granada y solicitamos permiso para acudir a clase con el fin de solicitar la colaboración de los estudiantes. Asimismo, para evitar sesgar los resultados se obvió el tema de la investigación, indicando únicamente el contexto académico de la misma. La composición del grupo de discusión que finalmente participó en el encuentro quedó configurada según se detalla en la Tabla 3.

\section{Guía de preguntas}

Como paso previo a la celebración del grupo de discusión, se elaboró una guía inicial de preguntas sobre las áreas temáticas de discusión. El diseño de la guía para el grupo de discusión se elaboró de acuerdo con tres criterios: la revisión de la literatura existente sobre el tema de estudio, los objetivos del presente trabajo y la ordenación gradual, desde lo general hacia lo específico. La
Tabla 4, que no incluye las preguntas surgidas en el transcurso de la reunión, fundamenta la guía de preguntas.

\section{Reducción de datos}

Una vez finalizada cada una de las sesiones, iniciamos la transcripción del discurso oral que, con el consentimiento de los informantes, fue grabado. En tanto que el análisis de los datos tiene como objetivo el contenido, es decir, las ideas, optamos por una transcripción literal sin edición. Por lo tanto, la fidelidad al modo de pensar y de expresarse de los informantes ha sido la pauta que ha marcado dicha labor, quedando subsumida toda consideración estilística y posible acercamiento al lector del discurso.

Tras la codificación de todas las transcripciones, clasificamos cada uno de los fragmentos 
344 representativos en diferentes secciones, atendiendo a las categorías temáticas establecidas. Para realizar dicha tarea se utilizó Atlas.ti, que permite recuperar citas y códigos con la identificación correspondiente al texto original. A continuación, iniciamos la reclasificación del material agrupado en cada sección para proceder a su inspección conjunta. En la categorización y codificación del material discursivo utilizamos un enfoque mixto: aunque las categorías fueron establecidas a priori, resultado de la revisión de la literatura sobre el tema de estudio y los objetivos de la investigación (procedimiento deductivo), también se identificaron nuevas categorías a las que se asignó un código una vez iniciado el análisis del material recopilado en el transcurso de las sesiones (procedimiento inductivo).

\section{Obtención de resultados y verificación de conclusiones}

De acuerdo con Valles (2003: 231), existen dos modos generales de orientar el análisis y la presentación de los datos: el análisis centrado en las cuestiones, temas o asuntos; y el análisis centrado en los casos estudiados. En este estudio nos hemos ceñido al primero de ellos, puesto que seguiremos el «esqueleto» de las baterías temáticas de preguntas que se siguieron durante la recogida de datos. Asimismo, el tipo de análisis e informe debe decidirse teniendo en cuenta los objetivos y circunstancias de cada investigación. Dado el propósito exploratorio de este estudio, una descripción narrativa suele resultar apropiada (Stewart y Shamdasani, 1990). A continuación, exponemos una síntesis de cada una de las categorías de análisis a las que acompañan algunos de los comentarios más ilustrativos vertidos por los informantes.

\section{DISCUSIÓN DE RESULTADOS}

\section{Direccionalidad: implicaciones para la prácti- ca de la traducción}

Según los datos obtenidos a través del grupo de discusión, los informantes perciben una relación de causalidad entre la direccionalidad de la traducción y la calidad de esta, motivo por el cual los informantes se sienten más vulnerables al traducir desde su lengua $\mathrm{A}$ hacia su lengua $\mathrm{B}:-[. .$.$] «el caso es que no eres nativo, no eres$ hablante de una lengua y, por tanto, nunca va a tener calidad. Y como no va a tener calidad, no puedes sentirte nunca cómodo» - $\mathrm{S} 4$. Dominar activamente y y poseer grandes destrezas para escribir en la lengua $B$, independientemente de la tipología textual, se considera un factor clave.

No obstante, dentro del grupo se manifiestan posiciones divergentes. Al menos la mitad de los informantes indican que, indistintamente de la direccionalidad, los lenguajes especializados o jergas sobre los que versen la traducción también juegan un papel decisivo a la hora de desempeñar esta tarea. En este sentido, se percibe que los conocimientos temáticos no siempre son simétricos en las lenguas de trabajo del traductor, lo que también tendría un impacto significativo sobre los índices de calidad de un texto producido en la dirección A-B: — «depende del texto porque siempre hay campos que dominas mejor que otros, bien porque te gustan más, te parecen más atractivos, simplemente tienes más experiencia o más conocimientos»- $\mathrm{S} 5$. Asimismo, se cuestiona que el conocimiento avanzado de la lengua A y la capacidad de producir textos de todo tipo en dicha lengua de trabajo sea una consecuencia directa de ser «nativo» de una lengua. Lo que supone que, a diferencia de la traducción hacia lenguas A, la traducción hacia lenguas B implicaría una orientación consciente 
hacia la documentación adicional y el desarrollo de destrezas y mecanismos de revisión y textos propios. Por ejemplo, una informante declara: — «creo que cuando vas a usar la B estás mucho más alerta, te fijas mucho más en detalles como puntuación, mayúsculas, minúsculas [...]. También tienes la falsa idea de que por ser tu idioma materno sabes todo» - S3.

Por otro lado, al analizar los significados atribuidos a las lenguas implicadas en el proceso de traducción, se revela que una mayoría percibe las lenguas A y las lenguas B como entidades opuestas: - «pienso que cada uno tiene una lengua en que se siente más cómodo. Tú puedes hablar perfectamente dos idiomas, pero pienso que tienes un idioma en que te sientes más cómodo» - S6. Sin embargo, un informante subraya la necesidad de cuestionar la dicotomía $\mathrm{A} / \mathrm{B}$ en un contexto social que, si tomamos como punto de referencia el sistema educativo, camina hacia el bilingüismo: — «hemos mitificado la lengua $\mathrm{A}$, porque nos han hecho creer que dominamos la lengua materna. Es un error» - S3. Otras opiniones, que se sitúan a medio camino entre las posturas mencionadas, apuntan a las limitaciones que supone generalizar y la necesidad de hacer referencia a casos concretos. En este sentido, se insiste en el carácter mudable de la competencia lingüística, tanto de la lengua $\mathrm{A}$ como de las lenguas B, a lo largo de la vida de los traductores, en función de los contextos personales y profesionales en los que se desarrollen. Por esta razón, algunas voces han expresado su preocupación porque la división entre lengua A/ lengua $B$ pueda entenderse como una limitación de sus capacidades: se sostiene que el traductor debe desarrollarse en ambas lenguas con el mismo nivel de objetivos formativos. Reproducimos en las siguientes líneas el testimonio de una informante: - «creo que es un error y una limita- ción que a los traductores nos dibujen una línea muy clara de B-A/A-B. El objetivo de alguien que no es filólogo, sino de alguien que es traductor, es ver la A y la B tan cerca, a un nivel tan parecido mentalmente, y a nivel de práctica y de sentimiento $[. ..] \gg-S 3$.

En cuanto a la direccionalidad de la traducción, la postura de los informantes del grupo de discusión es unánime: prefieren traducir hacia su lengua A. Entre los motivos señalados se destaca el tiempo que se debe dedicar a la autorrevisión y evaluación de la calidad del texto producido: - «siempre a la lengua materna $<$ la mayoría asiente $\gg$-S6. Por tanto, se alude a la rentabilidad del proceso de traducción. Si bien, análogamente, también todos los informantes sostienen que aceptarían un encargo real de traducción hacia algunas o todas sus lenguas B.

\section{La direccionalidad en la clase de traducción}

Las principales diferencias percibidas entre la clase de traducción B-A y la clase de traducción A-B, direcciones de traducción que tienen lugar en un espacio físico y temporal diferentes de acuerdo con su organización en el plan de estudios, radican tanto en la organización del aprendizaje como en el perfil docente que las imparte, «nativo» de la lengua de destino de la clase: A en traducción B-A y B en traducción A-B.

Por lo que respecta a la configuración de asignaturas diferenciadas en función de la direccionalidad del proceso traslativo, todos los informantes coinciden en que se trata de un motivo de organización de la docencia y que, a su vez, sirve para que los traductores en formación se preparen para cada secuencia de aprendizaje: — «a efectos prácticos, porque realmente es mucho más fácil que estar siempre en ambas direcciones [...]. Entras con otra actitud y otra preparación» —S3. 
En este sentido, la mayoría de los informantes valora de forma positiva esta división, puesto que considera que una clase de traducción sin direccionalidad previamente definida podría llegar a convertirse en una clase de lengua o de teoría de la traducción. No obstante, al menos dos informantes apuntaron a la viabilidad de implementar este tipo de clase, en la que un mismo docente imparta la misma asignatura de práctica de la traducción organizando los contenidos de tal modo que se traduzca en direcciones diferentes a partir de encargos de traducción diferentes.

Igualmente, también se han señalado características contextuales propias del mercado de la traducción. Por ejemplo, un informante duda que los clientes crean que un traductor no puede traducir tanto hacia su lengua A como hacia su lengua B garantizando el mismo nivel de calidad. Y por añadidura, los clientes suelen confiar en el trabajo de los traductores a los que habitualmente solicitan su servicio sin reparar en la direccionalidad del proceso de traducción. Lo que, en suma, les hace pensar que tendrán que enfrentarse a dicha tarea si en algún momento se incorporan al mercado profesional de la traducción: - «precisamente por este motivo, nos vamos a tener que ver obligados a traducir en ambas direcciones» - $\mathrm{S} 4$.

En cuestión de organización, las clases de traducción han sido descritas de forma heterogénea en función del docente que imparte la asignatura e independientemente de la direccionalidad de la clase. Por ejemplo, se señala una variación significativa del tiempo que se dedica al desarrollo y explotación de los ejercicios previos a la traducción, en comparación con la dedicación a la propia tarea de traducción. No obstante, dicha falta de unicidad en cuestiones metodológicas se percibe como positiva por la mayoría de los informantes, que consideran que la interacción con diferentes docentes puede enriquecer su formación; en concreto, para la asimilación de diferentes tipos de estrategias y técnicas de traducción. En este sentido, las diferencias metodológicas existentes entre B-A/A-B han sido justificadas con base en la necesidad de que el alumno desarrolle destrezas específicas según la lengua del texto meta:

— «Sí que podrían variar un poco las clases, en desarrollar mucho más esas destrezas a la hora de expresarte [...]. Porque lo que sí tenemos claro es que vamos a tener quizás bastantes menos problemas de comprensión, pero vamos a tener que hacer un esfuerzo añadido a la hora de reexpresar.»-S1

Al mismo tiempo, los informantes perciben que existen diferentes niveles de exigencia asociados a los objetivos formativos en función de la direccionalidad de la clase de traducción y consideran que la unificación de objetivos mínimos en ambas direcciones tendría efectos muy positivos para la adquisición de competencias: — «[...] ayudaría a veces para mejorar la B, porque si haríamos [sic] lo mismo, nos dedicaríamos con mucho esfuerzo a hacer las dos direcciones» - S6. Por otro lado, además de las competencias propias de cualquier clase de traducción, en clase de traducción A-B, sobre todo en los cursos iniciales, se pone de manifiesto la existencia de un elemento de aprendizaje de la lengua B que parece ser menor a medida que se avanza en la formación: - «no es clase de lengua, pero [...] estamos matando dos pájaros de un tiro, porque estamos refrescando los conocimientos lingüísticos de alguna manera» - $\mathrm{S} 5$.

\section{El docente de traducción}

Aunque intuimos que esta situación puede extrapolarse a la mayoría de los centros de formación del territorio nacional, en la Facultad 
de Traducción de la Universidad de Granada, el perfil contractual del profesorado universitario de traducción se ha descrito como académico e investigador, lo que significa que, aunque el personal docente tenga plena capacidad o formación docente, en el acceso al ejercicio docente se valora casi exclusivamente la capacidad investigadora.

Con independencia del marco normativo del sistema universitario español, las competencias del perfil del «buen» docente universitario de traducción que se nombraron en el transcurso del grupo de discusión son las siguientes:

I. Conocimientos lingüísticos

2. Conocimiento del área temática de la traducción (p. ej., científico-técnica)

3. Experiencia profesional en traducción

4. Formación académica en traducción

5. Formación didáctica

6. Competencias transversales: tecnologías aplicadas a la traducción

A continuación, se exponen las opiniones más destacadas vertidas a lo largo de la sesión en las competencias que se perciben como importantes o muy importantes.

\section{- Conocimientos lingüísticos:}

La lengua A del docente de traducción se percibe como uno de los pilares fundamentales del perfil docente. No obstante, mientras que la mayoría de los informantes subraya las virtudes que supone la presencia del «nativo» de la lengua de destino para la clase de traducción, al menos dos informantes destacaron las ventajas del «no nativo». Por ejemplo: — «si no es nativo quizás sea una persona que también se ha enfrentado a los mismos problemas a los que se está enfrentando el alumno y por lo tanto sabe cómo o qué camino cogió él» —-S1. Y aunque se reconoce que el esfuerzo en la preparación de la clase de traducción probablemente sea superior para el «no nativo», en las etapas iniciales de la formación se alude a las ventajas de una docencia más continuada a través de una persona que imparta las dos direcciones.

Otra de las implicaciones de la lengua A del docente de traducción podría manifestarse en el plano psicológico de la formación de traductores, que comprende la confianza en sí mismo. En tanto que el perfil del «nativo» promueve la superioridad de la traducción B-A en la propia clase de traducción A-B, resulta conveniente reflexionar sobre las implicaciones didácticas de la lengua A del docente de traducción A-B para el estudiantado. Aunque no tiene por qué generar desmotivación, es lógico asumir que el papel que adopte el docente resultará decisivo.

\section{-Experiencia profesional en traducción:}

La experiencia profesional es otro de los atributos sobre el que todos los informantes coinciden en considerar como muy importante. Se considera imprescindible para el desarrollo óptimo de la clase de traducción, llegándose a establecer una analogía deseable con el perfil del docente de interpretación, que, en la mayoría de los casos, está respaldado por una dilatada carrera profesional: — «para mí es fundamental un profesor de traducción e interpretación, o de traducción, o de interpretación, que tenga una experiencia previa como traductor o intérprete. Eso para mí es fundamental» — $\mathrm{S} 3$.

\section{-Formación académica:}

A diferencia de la experiencia profesional, en la formación académica del docente de traducción no se manifiesta una postura unánime. A pesar de que varios informantes coinciden en que la 
348 formación en traducción como disciplina académica sería idónea para ejercer la docencia, otros apuntan hacia un perfil más filológico $u$ otra titulación relacionada con el campo de especialidad de la traducción.

\section{-Formación didáctica:}

La formación didáctica continuada se constituye como otro de los atributos deseables del perfil docente. Todos los informantes mostraron una actitud crítica ante la necesidad de una formación inicial de los docentes noveles y una actualización continua del grupo de docentes veteranos. Reproducimos uno de los testimonios:

— «stá claro que tienes que saber traducir, pero una persona puede ser muy buena traduciendo, pero muy mala enseñando. Y entonces de poco puede servir que sea muy bueno traduciendo si no es capaz de transmitir el conocimiento al alumno y que el alumno sepa aplicar luego ese conocimiento.»-S5

\section{Satisfacción con la formación recibida en traducción A-B}

Para finalizar, en cuestión de satisfacción con la formación recibida en traducción A-B, todos los informantes se mostraron vacilantes y cautos con sus respuestas. Señalaron la dificultad de dar una respuesta genérica a dicha pregunta como resultado de la diversidad de metodologías y enfoques didácticos que han experimentado en diferentes asignaturas del plan de estudios. Si bien el grado de satisfacción en general es bueno, se destacan diferentes factores que intervienen en el nivel de satisfacción. Entre ellos, la figura del docente y, seguidamente, la motivación se distinguen como los más significativos. La mayoría de los informantes establecen un vínculo con el interés y el trabajo personal como elementos determinantes para la satisfacción con la formación recibida, tanto en traducción A-B como en $\mathrm{B}-\mathrm{A}:$ - «el que tiene interés intenta aprender más, intenta traducir mejor, aunque sea su lengua B. En mi caso, con mis profesores, yo diría que eso es lo que más peso tenía» —S1.

\section{Otras observaciones}

En otro orden de cosas, otro factor que se introdujo a debate fue el número de créditos existente en traducción A-B por oposición al de B-A. En este sentido, se detectaron tres posturas diferentes: la defensa del planteamiento actual, por entender que se trata de un tipo de traducción que un traductor profesional no debería abordar; el deseo de contar con un número de créditos análogo al de traducción B-A, que lamenta que haya asignaturas que solo se oferten en esta última modalidad (como la de traducción audiovisual) o solo en algunas combinaciones lingüísticas; y, por último, la necesidad de aumentar el número de créditos hasta el punto de que la carga lectiva sea superior a la de traducción B-A, por considerar que la traducción A-B es la más «problemática».

\section{A MODO DE CONCLUSIÓN}

Como objeto de estudio, la investigación de la traducción A-B ha favorecido la reflexión crítica sobre la superioridad de la traducción B-A desde una perspectiva teórica. En este artículo hemos señalado que las actitudes hacia la direccionalidad en traducción varían dependiendo de varios factores como la cultura de traducción, uno de los principales factores que determinan la actitud prescriptiva frente a la teoría y práctica de la traducción.

En la universidad española, la traducción A-B ha ocupado y todavía hoy ocupa un lugar 
marginal en la formación de traductores. Actualmente, si bien el Libro Blanco del nuevo título de Grado en Traducción e Interpretación reconoce entre los objetivos de formación «la competencia traductora general y especializada, tanto hacia la lengua propia como hacia al menos dos lenguas extranjeras», tras la reestructuración de los planes de estudio de acuerdo con el EEES la traducción A-B ha dejado de ser una materia básica o contenido de obligatoria inclusión, como lo fuera en los estudios conducentes a la Licenciatura en Traducción e Interpretación. Todo ello tiene a su vez implicaciones para otro asunto: quién enseña a traducir. Por otra parte, esta es otra premisa que subyace a la cuestión de la direccionalidad en la formación de traductores: el docente de traducción y, quizá en menor grado de interpretación, debe ser «nativo» de la lengua de destino de la clase.

En este artículo hemos presentado un estudio exploratorio-descriptivo en el que participaron seis traductores en formación - cuatro estudiantes permanentes y dos estudiantes de intercambio-de los dos últimos cursos del Grado de Traducción e Interpretación de la Universidad de Granada. El instrumento de recogida de datos elegido fue el grupo de discusión, una técnica de investigación cualitativa que resulta especialmente conveniente para identificar los valores atribuidos por un grupo social — los traductores en formación - a un fenómeno - la direccionalidad de la traducción.

De acuerdo con el análisis de los datos obtenidos, la mayoría de los traductores en formación perciben las lenguas A y B como entidades opuestas. Sin embargo, también se ha subrayado la necesidad de cuestionar la dicotomía A/B en referencia al carácter mudable de la competencia lingüística. Se considera que las lenguas A, B y C pueden cambiar a lo largo de la vida del traductor en función de contextos personales y profesionales. Asimismo, se ha cuestionado que el conocimiento avanzado de la lengua A y la capacidad de producir textos de todo tipo en dicha lengua de trabajo sea una consecuencia directa de ser «nativo» de una lengua.

Por otra parte, los traductores en formación perciben que, si bien la direccionalidad de la clase no entraña per se una modificación de la metodología didáctica para la enseñanza de la traducción, en la clase de traducción A-B existe un elemento de enseñanza y aprendizaje de la lengua B. Con referencia a los objetivos de formación, juzgan que la unificación de objetivos mínimos en las clases de traducción B-A y A-B, les permitiría abordar tareas para la adquisición de una competencia traductora plena y suficiente para incorporarse eficazmente al mercado laboral de la traducción y enfrentarse con garantías a los procesos de traducción.

En lo que respecta al docente de traducción y su perfil lingüístico, se manifiestan dos posturas opuestas: para algunos, la lengua A del docente es uno de los factores determinantes para el éxito de la formación de traductores; para otros, sin embargo, otro tipo de competencias, como la experiencia profesional, deben recibir especial atención.

Por último, acerca de la valoración de la carga lectiva en traducción A-B, se observan tres posturas divergentes: la defensa del planteamiento actual; el deseo de contar con un número de créditos análogo al de traducción B-A; y el ruego de aumentar el número de créditos hasta el punto de que la carga lectiva sea superior a la de traducción B-A.

A modo de valoración global, aunque somos conscientes de que las conclusiones extraídas de este estudio exploratorio no son extensibles al resto de la población de traductores en forma- 
ción que cursan sus estudios en universidades españolas, sí que podríamos hablar de ciertas tendencias que invitan a continuar investigando para ampliar la línea de direccionalidad de la traducción en el contexto de la formación de traductores.

\section{REFERENCIAS BIBLIOGRÁFICAS}

Alves Ferreira, Aline (2012). «Investigando o processamento cognitivo de tradutores profissionais em tradução direta e inversa no par linguístico inglêsportuguês», Cadernos de Tradução, 29 (1), 73-92.

BeEby Lonsdale, Allison (2009): «Directionality», en Mona Baker y Gabriela Saldhana (eds.). Routledge Encyclopedia of Translation Studies, Londres: Routledge, 84-88.

BeEby LonsDALE, Allison (1996): Teaching Translation from Spanish to English: worlds beyond words, Otawa: University of Ottawa.

BranchADELL, Albert (2012): «Minority languages and translation», en Yves Gambier y Luk van Doorslaer (eds.) Handbook of Translation Studies (2), Amsterdam y Philadelphia: John Benjamins Publishing Company, 97-101.

Buendía, Leonor, María Pilar Colás y Fuensanta Hernández (1998): Métodos de investigación en psicopedagogía, Madrid: McGraw-Hill.

Camprell, Stuart (1998): Translation into the Second Language, London/New York: Longman.

CHodkiewicz, Marta (2016): «What types of errors do undergraduate students make depending on directionality?» en Lukasz Bogucki, Barbara Lewandowska-Tomaszczyk y Marcel Thelen (eds.) Translation and meaning - New series, 2(2), Frankfurt am Main: Peter Lang, 191-208.

Cómitre Narváez, Isabel (2003): «La enseñanza de la traducción inversa (español-francés): realidad profesional y desafío didáctico», en Ricardo Muñoz Martín (ed.) I AIETI. Actas del I Congreso Internacional de la Asociación Ibérica de Estudios de Traducción e Interpretación, Granada 12-14 de Febrero de 2003. Granada: AIETI, 2, 383-389. <http://www.aieti.
eu/wp-content/uploads/AIETI_1_ICN_Ensenanza. pdf> [consulta: 06-VIII-2018].

Contreras García, Lucía y Miguel Turrión (2013): «Inversa traducción en la Europea Comisión», $P a-$ nace@, 14 (38) 265-274. <http://www.medtrad. org/panacea/PanaceaPDFs/Panacea38_Diciembre2013.pdf> [consulta: 06-VIII-2018]

Cruz Trainor, María M. de la (2004): «Traducción inversa: una realidad», TRANS. Revista de Traductología, 8, 53-60. <http://www.trans.uma.es/Trans_8/ t8_53-60_MDeLaCruz.pdf> [consulta: 06-VIII2018].

DA SILVA, Igor Lourenço; Fabio Alves; Márcia Schmaltz; Adriana Pagano; Derek Wong; Lidia Chao, y Gabriel Eduardo da Silva (2017): «Translation, postediting and directionality», en A. L. Jakobsen y B. Mesa-Lao (eds.), Translation in Transition: Between cognition, computing and technology, 108-134.

De Lima FonseCA, Norma Barbosa (2015): «Directionality in translation: Investigating prototypical patterns in editing procedures», Translation \& Interpreting, 7 (1), 111-125.

Davies, Alan (2003): The Native Speaker: Myth and Reality, Clevendon: Multilingual Matters.

FerReIRA, Aline; John W. Schwieter; Alexandra Gottardo, y Jefferey Jones (2016): «Cognitive effort in direct and inverse translation performance - Insight from eye-tracking technology», Cadernos de Tradução, 36 (3), 60-80.

Gallego Hernández, Daniel (2014a): «A vueltas con la traducción inversa especializada en el ámbito profesional - Un estudio basado en encuestas», TRANS. Revista de Traductología, O (18), 229-238. <http://www.trans.uma.es/Trans_18/ Trans18_227238_notas.pdf> [consulta: 06-VIII2018].

Gallego Hernández, Daniel (2014b): «Didáctica de la traducción inversa especializada - Propuesta de actividades en el ámbito de la economía y los negocios», LSP Journal, 5(1), 79-99. <https://rauli. cbs.dk/index.php/lspcog/article/view/4281> [consulta: 06-VIII-2018].

Grosman, Meta; Mira Kadric; Irena Kovaciiĉ, y Mary Snell-Hornby (eds.) (2000): Translation into Non- 
mother Tongues in Professional Practice and Training, Tubinga: Stauffenburg.

HAGEMANN, Susanne (2019): «Directionality in translation and revision teaching: a case study of an A-B teacher working with B-A students», The Interpreter and Translator Trainer, 13 (1), 86-101.

HorCAs-RuFiÁn, Sara y Dorothy Kelly (2020): «Inverse $(\mathrm{A}-\mathrm{B} / \mathrm{C})$ translation education in Spain under the EHEA», Perspectives, 28 (2), 300-319, DOI: 10.1080/0907676X.2019.1684529.

Hurtado AlbiR, Amparo (2001): Traducción y traductología: introducción a la traductología, Madrid: Cátedra.

IAPTI (2015): «Translation into a non-native language». <https://www.iapti.org/files/surveys/2/ IAPTI_non-native_report.pdf> [consulta: 06-VIII2018]

ITI - Institute of Translation and Interpreting (2012): Code of professional conduct: individual members. <http://www.iti.org.uk/attachments/article/154/ Code\%20of\%20Conduct\%20-\%20individual.pdf> [consulta: 06-VIII-2018]

KelLy, Dorothy (1997): «La enseñanza de la traducción inversa de textos "generales". Consideraciones metodológicas», en Miguel Ángel Vega y Rafael Martín-Gaitero (eds.) La palabra vertida - Investigaciones en torno a la traducción: Actas de los VI Encuentros Complutenses en torno a la traducción, 175-181. Madrid: Ediciones del Orto <http://cvc. cervantes.es/lengua/iulmyt/pdf/palabra_vertida/18_kelly.pdf> [consulta: 06-VIII-2018]

KelLy, Dorothy (2003): «La investigación sobre formación de traductores: algunas reflexiones y propuestas», en Emilio Ortega Arjonilla (dir.) $P a$ norama actual de la investigación en traducción e interpretación, 1 (3), 585-596. Granada: Atrio.

Kelly, Dorothy (2005). «The Wrong Way Round? Consideraciones sobre la cuestión de la direccionalidad en la traducción profesional y la formación de traductores», en Cristina García de Toro e Isabel García Izquierdo (eds). Experiencias de traducción. Reflexiones desde la práctica traductora, Castelló de la Plana: Servicio de Publicaciones de la Universitat Jaume I, 129-146.
Kelly, Dorothy; Marie-Louise Nobs, Dolores Sánchez y Catherine Way. (eds.) (2003): La direccionalidad en Traducción e Interpretacion: perspectivas teóricas, profesionales y didácticas, Granada: Atrio.

KIRALY, Don C. (1995): Pathways to Translation. Pedagogy and Process, Kent/Ohio: Kent State.

Krueger, Richard A. (1988). Focus groups: A practical guide for applied research, Thousand Oaks: Sage $\mathrm{Pu}$ blications.

Ley Orgánica 15/1999, de 13 de diciembre, de Protección de Datos de Carácter Personal (BOE núm. 298, de 14 de diciembre de 1999, 43088-43099). $<$ https://www.boe.es/buscar/doc.php?id=BOEA-1999-23750> [consulta: 06-VIII-2018].

Lıс̌ко, Roman (2014): Translation into English as a Foreign Language. A Slovak Survey, tesis doctoral, Matej Bel University

Martínez Melis, Nicole (2001) Évaluation et didactique de la traduction - Le cas de la traduction dans la langue étrangère, tesis doctoral, Universidad Autónoma Barcelona.

Mayoral Asensio, R. (2001). «Por una renovación en la formación de traductores e intérpretes: revisión de algunos de los conceptos bore los que se basa el actual sistema, su estructura y contenidos», Sendebar, 12, 311-336.

McMillan, James y Sally Schumacher (2005): Investigación Educativa. Una introducción conceptual, Madrid: Pearson Addison Wesley

NidA, Eugene A. y Charles Russell Taber (1982): The Theory and Practice of Translation, Leiden: The United Bible Society, E. J. Brill.

Opdenhoff, Jan (2011): Estudio sobre la direccionalidad en interpretación de conferencias: de la teoría a la práctica profesional, tesis doctoral, Universidad de Granada.

PAvlović, Natasa (2007): Directionality in Collaborative Translation Processes: a Study of Novice Translators, tesis doctoral, Universitat Rovira i Virgili.

PAvlović, Natasa y Kristian Jensen (2009). «Eye tracking translation directionality», en Anthony Pym y A. Perekrestenko (eds.), Translation Research Projects, 2, 93-109. Tarragona: Intercultural Studies Group.

Pavlović, Natasa (2010): «What were they thinking?! 
Students decision making in L1 and L2 translation processes», Hermes, Journal of Language and Communication Studies, 44, 63-87. <http://download2. hermes.asb.dk/archive/download/Hermes-44-pavlovic.pdf> [consulta: 06-VIII-2018]

Pietrzak, Paulina (2013): «Divergent Goals: Teaching Language for General and Translation Purposes in Contrast», en Piątkowska Katarzyna y Ewa Kościałkowska-Okońska (eds.) Correspondences and Contrasts in Foreign Language Pedagogy and Translation Studies. Second Language Learning and Teaching. Heidelberg: Springer.

РокоRN, Nike (2005): Challenging the Traditional Axioms. Translation into a Non-Mother Tongue, Ámsterdam-Filadelfia: John Benjamins.

PокоRN, Nike (2009): «Natives or Non-natives? That is the Question...Teachers of Translation into Languages B», The Interpreter and Translator Trainer, 3 (2), 189-208.

PокоRN, Nike (2016): «Is it so different? Competences of teachers and students in L2 translation classes», Rivista internazionale di tecnica della traduzione,18, 31-48. <https://www.openstarts.units. it/bitstream/10077/13664/1/Pokorn_Ritt_18_2016. pdf> [consulta: 06-VIII-2018]

PRUNČ, Erich (1997): «Translationskultur (Versuch einer konstruktiven Kritik des translatorischen Handelns)», TextconText, 11 (2), 99-127.

Prunč, Erich (2003): «Óptimo, subóptimo, fatal: Reflexiones sobre la democracia etnolingüística en la cultura europea de traducción», en Dorothy Kelly, Marie-Louise Nobs, Dolores Sánchez y Catherine Way. (eds.) La direccionalidad en Traducción e Interpretacion: perspectivas teóricas, profesionales y didácticas, Granada: Atrio, 67-89.

Real Decreto 1385/1991, de 30 de agosto, por el que se establece el título universitario oficial de Licenciado en Traducción e Interpretación y las directrices generales propias de los planes de estudios conducentes a la obtención de aquel (BOE núm. 234, de 30 de septiembre de 1991, 31773-31775). <https://www.boe.es/buscar/doc.php?id=BOEA-1991-24111> [consulta: 06-VIII-2018].

Robinson, Douglas (1997): Western translation theory: from Herodotus to Nietzsche, Manchester: St. Jerome.
RodríGuez, Nadia y Bettina Schnell (2012): «Direccionalidad y formación de traductores - Un estudio longitudinal de los procesos cognitivos en la traducción inversa», Meta: journal des traducteurs / Meta: Translators' Journal, 57 (1), 67-81.

Rodríguez-InÉs, Patricia (2008). Uso de corpus electrónicos en la formación de traductores (inglés-españolinglés), tesis doctoral no publicada, Universidad Autónoma de Barcelona.

RoIss, Silvia (2001): «El mercado de la traducción inversa en España. Un estudio estadístico», Hermēneus, 3, 397-408.

RoIss, Silvia (2008): Desarrollo de la competencia traductora - Teoría y práctica del aprendizaje constructivo: Traducción inversa, español-alemán, Granada: Comares.

Stewart, David W. y Prem N. Shamdasani (1990). Focus groups Theory and practice. Applied social research methods series, Thousand Oaks: Sage Publications.

SuÁrez Ortega, Magdalena (2005): El grupo de discusión: Una herramienta para la investigación cualitativa, Barcelona: Laertes.

VALLEs, Miguel S. (2003): Técnicas cualitativas de investigación social. Reflexión metodológica y práctica professional, Madrid: Síntesis.

Wimmer, Stefanie (2011): El proceso de la traducción especializada inversa: modelo, validación empírica y aplicación didáctica, tesis doctoral, Universidad Autónoma de Barcelona.

Woodsworth, J. (1998): «History of Translation», en Mona Baker (ed.) Routledge Encyclopedia of Translation Studies, Londres-Nueva York: Routledge, 100-105.

Zimmermann, Petra (2007): «Misión (casi) imposible. La traducción especializada inversa al alemán desde la mirada del alumno», en Belén Santana, Silvia Roiss y María Ángeles Recio (eds.) Puente entre dos mundos: últimas tendencias en la investigación traductológica alemán-español, Actas del III STIAL, Universidad de Salamanca, 394-402. 